\title{
Hospital Based Study of Traumatic Neck Injuries: A Decision Making Approach
}

https://doi.org/10.47210/bjohns.2020.v28i2.320

\section{Abdullah Musleh ${ }^{1}$}

\begin{abstract}
Introduction
Traumatic neck injuries can cause serious morbidity and mortality. They have very serious outcomes and require emergent surgical interventions. This study illustrated 31 traumatic neck injury cases and their management over a period of 3 years. Materials and Methods

Thirty nine (39) patients were studied retrospectively and analyzed. Patients admitted through emergency room with a history suggestive of vascular trauma in the neck, between February 2014 and March 2017 were included. Thirty one (31) patients which were treated surgically were selected for analysis. All the patients were taken to the operating room immediately and made hemodynamically stable, other parameters were quickly corrected and urgent measurements were undertaken to maintain vascular integrity.

$\underline{\text { Results }}$

Out of 31 patients suspected to have vascular neck injuries, only 29 (74\%) were established to have vascular neck injuries. Forty seven percent $47 \%$ were stabilized hemodynamically and underwent operations under elective conditions. 16 (55\%) patients were found to be hemodynamically unstable with fair signs of vascular injuries and were taken to the operating room immediately. $41 \%$ had arterial injuries and $59 \%$ had venous injury. No patients had any post-operative vascular complication. One patient expired on the 11th postoperative day as a result of multiple organ injury and brain injuries.

Conclusion

Patients with severe neck injuries leading to acute bleeding and hematoma must be taken to the surgery for best outcome. Prompt decision making plays very important role to minimize disastrous consequences leading to further vascular and neurological derangements.

$\underline{\text { Keywords }}$

Neck Injuries; Vascular System Injuries; Decision Making
\end{abstract}

ABSTRACT

$\mathrm{N}$ eck injuries often involve airway compromise, potential spinal cord injuries and large amounts of blood loss from external wounds. ${ }^{1,2}$ All the patients with neck injuries should be taken seriously, and rapid transport to the closest appropriate facility should be ensured. The care provided in the field is extremely important for the patient and it increases survival. ${ }^{1,2}$

Blunt trauma has varied severity. It is always difficult to identify blunt trauma, but the injuries to the neck and

1 - Department of Surgery, College of Medicine, King Khalid University, Abha, KSA

\section{Corresponding author:}

Dr Abdullah Musleh

email: abdullahmuslehent@gmail.com spine can have significant consequences. These injuries require multimodal assessment. Spinal cord injury entails high level of concern as do fractures of the larynx and trachea, along with blunt injury to carotid arteries. ${ }^{1}$

Other injuries are penetrating neck injuries (PNIs) caused by gunshot wounds, stab wounds, or penetrating debris such as shrapnel. All PNIs are treated expectantly and mortality rates were high before World War II. $^{3}$ Aiming to reduce the high death rates associated with neck injuries, a mandatory surgical approach was adopted, even for stable patients to reduce mortality. ${ }^{4}$

Surgical exploration remained widely accepted as necessary well into the 1990 s, when it became obvious that while the mortality rate was low, the rate of negative surgical explorations was unacceptably high $(58 \%)$ and 
the concept of selective surgical management emerged with the advent of a spectrum of diagnostic modalities. ${ }^{5}$

PNIs, defined by platysma violation, comprise a significant number of traumatic injuries in adults and their treatment is trick. ${ }^{3}$ Approximately 15 to 20 percent of PNI cases required treatment. The management of a hemodynamically stable patient is still debatable ${ }^{3,6,7}$

Penetrating neck trauma may present with unpredictable signs and symptoms in the emergency. Around $5 \%$ to $10 \%$ of all neck injuries comprises of penetrating neck trauma and $30 \%$ of patients present with multiple injuries. Thorough knowledge of the anatomy of the neck, physical assessment, and current recommendations for diagnostic and therapeutic interventions are necessary for appropriate management. Express decision making is often required to prevent catastrophic airway, vascular, or neurologic sequelae.

Overall mortality attributed to neck trauma is $11 \%$. Injuries to the carotid or subclavian vessels bear the lion's share of such mortality and are generally associated with poorer outcome.

Injury to certain anatomic structures (e.g., the carotid or subclavian vessels) may be fatal in two thirds of cases. Surgeons show lack of consensus regarding which injuries warrant surgical intervention, especially in cases where conservative approach was taken primarily. 8

\section{Materials and Methods}

Thirty one (31) patients who were admitted through casualty room with a history of neck injury between Feb 2015 and March 2018 were analyzed by a team of otolaryngology surgeons. Twenty nine (29) of them were found to have vascular lesion. The necks of the other 2 patients were also explored, but no major vascular lesion was found.

Patients who presented with wounds in the neck and were admitted to our side but investigated and kept under observation for 24 hours without surgical interference were not included in our study.

All patients who were unstable were resuscitated according to Advanced Trauma Life Support protocol. Patients with heavy uncontrolled bleeding, expanding or pulsatile hematoma or no response to resuscitation were given prioritized for immediate surgery.

In patients with evidence of a vascular injury, the Glasgow Coma Score (GCS), systolic blood pressure (SBP) and positive neurological signs were recorded prior to surgical intervention. Neurological assessment was done pre and post operatively. Computerized axial tomography (CAT) scan of the brain was done in all the patients who were in coma for more than four hours duration with focal neurological signs.

Patients with injury to internal carotid artery and common carotid artery were taken to operation theatre immediately for repair even in coma or neurological insufficiency.

Seven patients whose conditions were stable underwent carotid artery Doppler ultrasonography.

Some patients posed difficult intubation through orotracheal route due to a big hematoma in the neck or difficulty in opening the mouth; trans-nasal intubation was performed in such patients using fibre-optic bronchoscope.

Arterial lesions were either repaired primarily or with venous graft interposition or ligated. Venous injuries were mostly ligated as all venous injuries were unilateral. Two patients with venous injuries were repaired primarily.

Patients were followed up at the outpatient clinic and duplex scan was done for those with arterial repairs.

\section{Results}

Twenty-nine patients, 21 males (72\%) and 8 females $(28 \%)$, found to have a vascular injury in the neck, were operated upon. Their ages ranged from 12-61 years with a mean of 41 years.

Sixteen patients (55\%), who were unstable at presentation to the ER with definite signs of vascular injury, were taken immediately to OR. Rest of the patients $(45 \%)$ were stabilized and evaluated by further investigation especially, duplex scan and or CTA.

Arterial injuries were found in 12 patients $(41 \%)$ and venous injuries in 17 patients (59\%).

Exploration was done through the anterior border of the sternocleidomastoid muscle. One patient also 
needed a left anterolateral thoracotomy for subclavian artery injury.

The common carotid artery was injured in $5(17 \%)$ patients. The internal carotid artery with the common carotid artery was injured in $3(10 \%)$ patients. The internal carotid artery was injured in $1(3 \%)$ patient. The external carotid artery was injured in 1 patient. The common, internal and external carotid arteries were found injured in $1(4 \%)$ patient. The subclavian was injured in $1(4 \%)$. Vertebral arteries were injured in 2 $(7 \%)$ patients.

Venous injury was mostly in the internal jugular vein (in 10 patients, i.e. $40 \%$ ). Four patients presented with injury to the external jugular vein.

Most of the vascular neck injuries were on the right side (19 patients, i.e. $66 \%$ ). The cause of the vascular injury was penetrating injury by gunshot, knife, sharp rod or iatrogenic during insertion of a permcath for hemodialysis. Other injuries (6 patients, i.e. $21 \%$ ) were associated with road traffic accidents, fall from a height or falling of heavy objects on the neck and shoulder.

Patients' morbidity was mainly due to bullet injury to carotid artery. There was no postoperative vascular bleeding, hematoma, thrombosis or infection in any patient treated for their vascular injuries.

Mortality was seen in one patient. He was a patient with multiple trauma, fall from a height with head fracture, left transverse cervical spine fracture, left vertebral artery injury, fracture ribs with lung contusion. He succumbed on the 12th post-operative day. The cause of death was suspected to be ARDS.

Routine follow up of the patients was done in the outpatient clinics. Duplex scan was done after 3 months for all the patients on whom arterial repair and revascularization. It showed patency of the arterial flow.

\section{Discussion}

Expeditious decision making is often required to prevent catastrophic airway, vascular, or neurologic sequelae. ${ }^{1,2,8}$

A large number of studies since the 1970's have attempted to determine the reliability of the physical exam in screening patients who should undergo further evaluation and treatment for penetrating neck injuries. Mandatory neck exploration for penetrating neck wounds often led to a negative exploration rate (in excess of $50 \%$ ). A review of the literature published in 1994 by McConnell et al. compares several studies that use physical examination to screen patients. The review notes that in those studies in which asymptomatic patients were placed in observation groups (serial q6 hour exams by a physician), the rate of negative neck exploration was low, while the rate of false negatives was negligible. ${ }^{4}$

Serious morbidity and mortality caused by carotid artery injuries must be intervened promptly and monitored closely after surgery. Arterial Doppler ultrasonography provides useful information in these injuries, conventional method being angiography of aorta visualizing its branches. ${ }^{9}$ However, patients with unstable condition, hematoma and uncontrolled bleeding were posted for immediate surgery. ${ }^{10}$ Usually young patients with neck injury were taken up for surgery, and arterial integrity was promptly restored leading to satisfactory outcome. Time elapsed between injury and surgery is a very important factor and it is evident that lesser time elapsed ensures better outcome.

Injury due to sharp objects show with cuts. In blunt injury, the surface may not reveal anything, making examination and management difficult. Neck and spinal injuries should be considered in such types of blunt trauma. ${ }^{11}$

It will be useful in case of cervical injuries to examine and investigate the neck in three parts as described by Manson et al. in "discrimination zone criteria" of cervical injury. ${ }^{12}$

A routine surgical exploration should be performed in injuries around neck. Some surgeons and medical centres prefer to go for angiography with elective surgery.

In case of active bleeding, surgical exploration should be done electively.

In patients of penetrating trauma especially in zone I or II who have sufficient clinical evidence of a vascular injury and are hemodynamically unstable, an immediate oblique incision along with anterior border of sternocleidomastoid muscle should be considered for 
exploration. $^{13}$

In patients with a gunshot wound in zone II with a hematoma, an unidentified level of a carotid-jugular arteriovenous damage, lost carotid pulse without a neurologic deficit, or proximity to the vertebral artery, diagnostic tests are indicated. ${ }^{14}$ Current diagnostic options include duplex ultrasound, computed tomography (CT) angiography, digital subtraction arteriography, and magnetic resonance angiography. In most of the hospitals, arteriography remains the gold standard to explore carotid artery injuries. ${ }^{14}$ Duplex scanning has limitations for cervical wounds with large, soft tissue insults. CT angiography could be a better choice as a noninvasive investigation vis-a-vis conventional arteriography. A potential disadvantage is the degradation of image quality due to artifacts produced by metallic fragments embedded in the soft tissue. $^{15}$

For penetrating injuries, the absence of hard signs rules out surgically significant vascular injury. ${ }^{16}$

Studies that examine penetrating trauma to all zones of the neck show consistently that zone 3 injuries are the least likely to occur and the most difficult to treat surgically. ${ }^{17-19}$

The availability and accuracy of angiography in most institutions since the 1970s have brought about the concept of "selective management," which has become standard in some trauma centers for hemodynamically stable patients. ${ }^{15,20}$

This approach results in many negative angiograms, as well as positive studies that are not surgically significant. ${ }^{21,22}$

Liekweg and Greenfield recommended a reconstruction of the injured vessel in a review of 170 patients with isolated injury to the internal carotid artery or common carotid artery with normal neurologic examinations before surgery. After revascularization, $5 \%$ of the patients had neurologic deficit versus $33 \%$ in patients where the injured carotid artery was ligated $(p<0.002) .{ }^{23}$ Ramadan et al. reported an even more pronounced difference in neurologic deficit after vascular repair compared with ligation in neurologic asymptomatic patients $(8 \%$ vs $50 \%, \mathrm{p}<0.05) .{ }^{24}$ Other reports concluded that restoration should always be performed in the case of neurologically asymptomatic patients. ${ }^{25}$

The most important complication of a ligated internal carotid artery or an untreated internal carotid artery occlusion is that it may develop embolic sequelae. ${ }^{13} \mathrm{We}$ believe ligation should be reserved for (1) those patients who had prolonged coma for more than 4 to 6 hours, (2) technical reasons where surgical revascularization or endovascular repair is not possible, and (3) injuries to the external carotid artery and its branches. ${ }^{26}$

As a non-surgical treatment, watchful waiting is the only option. Some authors suggest systemic heparinization for proven occlusion of the carotid artery due to gunshot injury to neck without involving brain or other body parts. ${ }^{2}$

These recommendations are based on available data from patients with blunt carotid injuries with identical injuries in which heparinization has been observed to be linked to improvements in neurologic condition and survival. However, the role of anticoagulants in relation to safety and efficacy in the management of stroke following carotid injury has not been established. ${ }^{28}$

\section{Conclusion}

Excellent knowledge of anatomy of the neck, proper systematic physical evaluation and current and updated recommendation-based utilization of appropriate modalities of intervention are necessary for management. Rapid decision-making capability and skill of a surgeon are required to prevent further deterioration of vascular, airway and neurologic status.

Patient with serious bleeding and in unstable condition with hematoma should be taken to surgery immediately and managed by multidisciplinary approaches. Stable patients with suspected vascular injury can be planned for elective surgery after performing in-depth examination, Doppler ultrasonography and CTA. Reconstruction of the arterial and venous injury shows the best outcome in most of the patients.

\section{References}

1. Burda TM, Cotton BA. Straight for the jugular: managing blunt 
\& penetrating neck trauma in the field. JEMS. 2007;32(8):40-9. doi:10.1016/S0197-2510(07)72293-3

2. American College of Surgeons Committee on Trauma Advanced Trauma Life Support for Doctors: Student Course Manual, 2004 7th ed. American College of Surgeons: Chicago

3. Thal ER, Meyer DM. Penetrating neck trauma. Curr Probl Surg. 1992; 29: 1

4. McConnell DB, Trunkey DD. Management of penetrating trauma to the neck. Adv Surg. 1994;27:97-127

5. Apffelstaedt JP, Muller, R. Results of mandatory exploration for penetrating neck trauma. World J Surg. 1994; 18:917

6. Asensio JA, Valenziano CP, Falcone RE, Grosh JD. Management of penetrating neck injuries. The controversy surrounding zone II injuries. Surg Clin North Am. 1991; 71(2):267-296. doi:10.1016/s0039-6109(16)45379-x

7. Bryant AS, Cerfolio RJ. Esophageal trauma. Thorac Surg Clin 2007;17(1):63-72. doi:10.1016/j.thorsurg.2007.02.003

8. Rezende-Neto J, Marques AC, Guedes LJ, Teixeira LC. Damage control principles applied to penetrating neck and mandibular injury. J Trauma. 2008;64(4):1142-1143. doi:10.1097/ TA.0b013e318166d2d29.

9. Montalvo BM, LeBlang SD, Nunez DB Jr, Ginzburg E, Klose KJ, Becerra JL, Kochan JP. Color Doppler sonography in penetrating injuries of the neck. AJNR Am J Neuroradiol.1996; 17(5):943-51

10. Sekharan J, Dennis JW, Veldenz HC, Miranda F, Frykberg ER. Continued experience with physical examination alone for evaluation and management of penetrating zone 2 neck injuries: results of 145 cases. J Vasc Surg 2000; 32

11. Martin MJ, Mullenix PS, Steele SR, Asensios A, Anderson CA, Demetriudes D, Salim A. Functional outcome after blunt and penetrating carotid artery injuries :analysis of the national trauma data bank. J trauma 2005; 59(4):860-4; 5/22/2012

12. Manson DO, Saletta JD, Freeark RJ. Carotid vertebral trauma. J Trauma 1969; 9: 987-99

13. Feliciano DV. Management of penetrating injuries to carotid arterv. World J Surg. 2001; 25(8):1028-35

14. Roon AJ, Christensen N. Evaluation and treatment of penetrating cervical injuries. J Trauma 1979; 19: 391-7

15. van $A s A B$, van Deurzen DF, Verleisdonk EJ. Gunshots to the neck: selective angiography as part of conservative management. Injury 2002; 33(5):453-6. doi:10.1016/s00201383(02)00056-6

16. Dennis JW, Frykberg EF, Veldenz HC, et al. Validation of nonoperative treatment of occult vascular injuries and accuracy of physical examination alone in penetrating extremity trauma: 5 to 10 year follow-up. J Trauma 1998; 44:243-53

17. Gerst PH, Sharma SK, Sharma PK. Selective management of penetrating neck trauma. Am .Surg. 1990; 56:553-5

18. Cohen ES, Breaux CW, Johnson PN, et al. Penetrating neck injuries: experience with selective management. South Med J. $1987 ; 80: 26-8$

19. Sclafani SJ, Panetta T, Goldstein AS, Phillips TF, et al. The management of arterial injuries caused by penetration of zone Ill of the neck. J Trauma 1985; 25:871-9

20. Bumpous JM, Whitt PI, Ganzel TM, McClane SD. Penetrating injuries of the visceral compartment of the neck. Am J Otolaryngol. 2000; 21:190-4

21. Azuaje RE, Jacobson LE, Glover J, et al. Reliability of physical examination as a predictor of vascular injury after penetrating neck trauma. Am Surg. 2003; 69(9):804-7

22. Nason RW, Assuras GN, Gray PR, et al. Penetrating neck injuries: analysis of experience from a Canadian trauma centre. Can J Surg. 2001:44: 122-6

23. Liekweg WG, Greenfield LJ. Management of penetrating carotid arterial injury. Ann Surg.1978; 188:587-92

24. Ramadan F. Rutledge R, Oiler D. Howell P, Baker C, Keagy R. Carotid artery trauma: a review of contemporary' trauma center experiences. Vasc Surg. 1995; 21:46-56

25. Nunez DB, Ibrres-Leon M, Munera F. (2004): Vascular injuries of the neck and thoracic inlet: helical CTangiographic correlation. Hadiog Taphles 2004; 24:1087-1100

26. Rogier H, J. Kropman, Jean-Paul P. M. de Vries, and Michiel J. M. Segers. Surgical Repair of a Gunshot Injury to the Left Carotid Artery: Case Reportand Review of Literature. Vascular and Endovascular Surgery 2008; 42 (2): 180-3

27. Biffl WL, Moore EE, Ryu RK, et al. The unrecognized epidemic of blunt carotid arterial injuries: early diagnosis improves neurologic outcome. Ann Surg. 1998; 228(4):462-70. doi:10.1097/00000658-199810000-00003

28. Hacke W, Stingele R, Steiner T, Schuchardt V, Schwab S. Critical care of acute ischemic stroke. Intensive Care Med. 1995; 21(10):856-62. doi:10.1007/BF01700973. 